\title{
Neutron stars in Einstein- $\Lambda$ gravity: the cosmological constant effects
}

\author{
G. H. Bordbar ${ }^{1,2 *}$, S. H. Hendi ${ }^{1,3 \dagger}$ and B. Eslam Panah ${ }^{1 \ddagger}$ \\ 1 Physics Department and Biruni Observatory, College of Sciences, Shiraz University, Shiraz 71454, Iran \\ ${ }^{1}$ Center for Excellence in Astronomy \& Astrophysics of Iran (CEAAI-RIAAM)- Maragha, IRAN, P.O. Box: 55134-441, Iran \\ ${ }^{3}$ Research Institute for Astronomy and Astrophysics of Maragha (RIAAM), P.O. Box 55134-441, Maragha, Iran
}

\begin{abstract}
Regarding a $d$-dimensional spherically symmetric line element in the context of Einstein- $\Lambda$ gravity, the hydrostatic equilibrium equation of stars is obtained. Then, by using the lowest order constrained variational (LOCV) method with the AV18 potential and employing microscopic many body calculations in the modern equation of state, the structure properties of neutron stars are investigated. Regardless of cosmological point of view and considering arbitrary positive and negative values of the cosmological constant, the maximum mass of the neutron stars and their corresponding radius in 4-dimensions are computed. The results show that there is an upper limit for the maximum mass of neutron star for positive cosmological constant $\left(M_{\max } \leq 1.68 M_{\odot}\right)$. On the other hand, it is shown that the Einstein gravity cannot explain the structure of neutron star with negative $\Lambda$. Other properties of neutron stars such as; the Schwarzschild radius, average density, compactness and Buchdahl- Bondi bound are studied. In addition, by using the Buchdahl-Bondi bound for neutron stars, stability of these stars is investigated. Finally, the dynamical stability is investigated and shown that the neutron stars follow the dynamical stability in this gravity.
\end{abstract}

\section{INTRODUCTION}

Most of the solar system phenomena such as the Mercury precession can be successfully explained in the context of Einstein gravity, but when we intend to study beyond the solar system or strong gravity regimes, this theory encounters some problems. Accelerated expansion of our universe is one of the main challenging subjects of cosmologists [1-3], whereas Einstein gravity cannot properly explain it. In order to interpret this expansion, some various candidates have been proposed by many authors. Among various alternatives, one may point out the modified gravities like Lovelock gravity [4 7], brane world cosmology [8 10], scalar-tensor theories [11 20] and $F(R)$ gravity [21 31].

From the cosmological point of view, the dark energy is an unknown form of energy which is hypothesized to permeate the spacetime, tending to accelerate the expansion of the universe [32]. The dark energy is the most accepted hypothesis to explain the observations since the 1990s indicating that the universe is expanding at an accelerating rate. Another model of these interesting candidates is related to the cosmological constant. In this theory, the cosmological constant may add to the Einstein Lagrangian [33, 34] for explaining such acceleration. The cosmological constant is the simplest possible form of dark energy, which leads to the current standard model of cosmology known as the $\Lambda$-CDM model and provides a good fit to many cosmological observations.

From the astrophysical point of view, stars reach the equilibrium state due to the balance between gravitational force and internal pressure. Therefore, in order to study the structure of stars, we should obtain a suitable hydrostatic equilibrium equation (HEE) and then we can study the structure properties of stars. The neutron and quark stars are in the category of celestial objects with high mass, high density and small radius, and therefore, they are called compact objects. Due to this fact, we need to take into account the effects of general relativity (spacetime curvature) for investigating their structures. The first HEE of stars in 4-dimensional Einstein gravity has been studied by Tolman, Oppenheimer and Volkoff (TOV) [35 337]. Also, the physical characteristics of stars using TOV equation have been investigated by many authors [38 45]. Recently, survey of compact objects in different gravity has attracted the attention of researchers, in particular, the models of modified gravity which involve higher curvature terms or a scalar field [46 [56]. On the other hand, if one is interested to study the structure and evolution of stars in different theories of gravity with various sources, the new HEE must be obtained. Therefore, in recent years, the generalizations and modifications of TOV equation have been investigated in various theories of gravity, such as: dilaton gravity [56], gravity's rainbow [57], massive gravity [58], $F(R)$ and $F(G)$ gravities [61? -64] and p ost-Newtonian theory [65] (see also Refs. [66 79] for more details).

The outline of our paper is as follows. In the next section, we consider a spherical symmetric metric and obtain the HEE in the Einstein- $\Lambda$ gravity for arbitrary dimensions $(d \geq 3)$. Then, we investigate stability and energy conditions for the equation of state of neutron star matter in 4-dimensions. Also, we study effects the cosmological constant on

\footnotetext{
* email address: ghbordbar@shirazu.ac.ir

$\dagger$ email address: hendi@shirazu.ac.ir (corresponding author)

‡ email address: behzad.eslampanah@gmail.com
} 
maximum mass and its radius of the neutron stars. Next, we examine effects of the cosmological constant on other properties of neutron stars such as; Schwarzschild radius, average density, compactness, Buchdahl-Bondi bound and dynamical stability. We finish our paper with some closing remarks.

\section{HEE IN THE PRESENCE OF COSMOLOGICAL CONSTANT}

As first step, we regard $d$-dimensional Einstein- $\Lambda$ gravity to obtain its related HEE. The action of the Einstein gravity with the cosmological constant in arbitrary dimensions, $d$, is given by

$$
I_{G}=-\frac{1}{16 \pi} \int_{\mathcal{M}} d^{d} x \sqrt{-g}(R-2 \Lambda)+I_{\text {Matt }},
$$

where $R$ and $\Lambda$ are, respectively, the Ricci scalar and the cosmological constant in $d$-dimensions and $I_{M a t t}$ is the action of matter field. Varying the action (1) with respect to the metric tensor $g_{\mu}^{\nu}$, the equation of motion can be written as

$$
R_{\mu}^{\nu}+\frac{1}{2} R g_{\mu}^{\nu}+\Lambda g_{\mu}^{\nu}=K_{d} T_{\mu}^{\nu}
$$

where $K_{d}=\frac{8 \pi G_{d}}{c^{4}}, G_{d}$ is the gravitational constant in $d$-dimensions and define as $G_{d}=G V_{d-4}$, where $G$ denotes the four dimensional gravitational constant and $V_{d-4}$ is the volume of extra dimensions. Also, the constant $c$ is the speed of light in the vacuum. In addition, $R_{\mu}^{\nu}$ and $T_{\mu}^{\nu}$ are the symmetric Ricci tensor and energy-momentum tensors, respectively.

Here, we want to obtain the static solutions of Eq. (2). For this purpose, we assume a spherical symmetric spacetime in the following form

$$
d s^{2}=f(r) d t^{2}-\frac{d r^{2}}{g(r)}-r^{2} d \Omega_{k}^{2}
$$

where $f(r)$ and $g(r)$ are the unknown metric functions of radial coordinates and $d \Omega_{k}^{2}$ is the line element of unit $(d-2)$-dimensional sphere

$$
d \Omega_{k}^{2}=d \theta_{1}^{2}+\sum_{i=2}^{d-2} \prod_{j=1}^{i-1} \sin ^{2} \theta_{j} d \theta_{i}^{2}
$$

On the other hand, the energy-momentum tensor for a perfect fluid is

$$
T^{\mu \nu}=\left(c^{2} \epsilon+P\right) U^{\mu} U^{\nu}-P g^{\mu \nu}
$$

where $\epsilon$ and $P$ are density and pressure of the fluid which are measured by local observer, respectively, and $U^{\mu}$ is the fluid four-velocity. Using Eqs. (2) and (5) and the metric introduced in Eq. (3), we can obtain the components of energy-momentum for $d$-dimensions as follows

$$
T_{0}^{0}=\epsilon c^{2} \quad \& \quad T_{1}^{1}=T_{2}^{2}=T_{3}^{3}=\ldots=T_{d-1}^{d-1}=-P .
$$

We consider the metric (3) and Eq. (6) for the perfect fluid and obtain the components of Eq. (2) in the following forms

$$
\begin{aligned}
& K_{d} c^{2} r^{2} \epsilon=\Lambda r^{2}+\frac{d_{2} d_{3}}{2}(1-g)-\frac{d_{2}}{2} r g^{\prime}, \\
& K_{d} f r^{2} P=-\Lambda r^{2} f-\frac{d_{2} d_{3}}{2}(1-g) f+\frac{d_{2}}{2} r g f^{\prime}, \\
& 4 K_{d} f^{2} r P=-4 \Lambda r f^{2}-\frac{2 d_{3} d_{4}(1-g) f^{2}}{r}+2 d_{3}(g f)^{\prime} f-r g f^{\prime 2}+r\left[g^{\prime} f^{\prime}+2 g f^{\prime \prime}\right] f,
\end{aligned}
$$

where $d_{i}=d-i$ and also, $f, g, \epsilon$ and $P$ are functions of $r$. We note that the prime and double prime are, respectively, the first and second derivatives with respect to $r$. 
Using Eqs. (7) - (9) and after some calculations, we obtain

$$
\frac{d P}{d r}+\frac{\left(c^{2} \epsilon+P\right) f^{\prime}}{2 f}=0
$$

Now, we obtain $f^{\prime}$ from Eq. (8) as follows

$$
f^{\prime}=\frac{2\left[r^{2}\left(\Lambda+K_{d} P\right)+\frac{d_{2} d_{3}}{2}(1-g)\right] f}{r g d_{2}} .
$$

Then, we calculate $g(r)$ by using Eq. (7) with the following form

$$
g(r)=1+\frac{2 \Lambda}{d_{1} d_{2}} r^{2}-\frac{c^{2} K_{d} M \Gamma\left(\frac{d_{1}}{2}\right)}{d_{2} \pi^{d_{2} / 2} r^{d-3}},
$$

where $M=\int \frac{2 \pi^{d_{1} / 2}}{\Gamma\left(d_{1} / 2\right)} r^{d_{2}} \epsilon(r) d r$ and $\Gamma$ is the gamma function, in which $\Gamma(1 / 2)=\sqrt{\pi}, \Gamma(1)=1$ and $\Gamma(x+1)=x \Gamma(x)$.

By considering Eqs. (11) and (12), and inserting them in Eq. (10), we can extract the HEE of Einstein- $\Lambda$ gravity in $d$-dimensions as

$$
\frac{d P}{d r}=\frac{\left[\frac{d_{1} d_{3} \Gamma\left(\frac{d_{1}}{2}\right)}{4 \pi^{d_{1} / 2}} c^{2} K_{d} M+r^{d_{1}}\left(\Lambda+\frac{d_{1}}{2} K_{d} P\right)\right]}{r\left[-\Lambda r^{d_{1}}+d_{1}\left(\frac{\Gamma\left(\frac{d_{1}}{2}\right)}{2 \pi^{d_{1} / 2}} c^{2} K_{d} M-\frac{d_{2}}{2} r^{d_{3}}\right)\right]}\left(c^{2} \epsilon+P\right),
$$

where for 4-dimensional case $(d=4)$, Eq. (13) reduces to the following equation

$$
\frac{d P}{d r}=\frac{\left[3 c^{2} G M+r^{3}\left(\Lambda c^{4}+12 \pi G P\right)\right]}{c^{2} r\left[6 G M-c^{2} r\left(\Lambda r^{2}+3\right)\right]}\left(c^{2} \epsilon+P\right) .
$$

Also, Eq. (14) leads to primitive TOV equation for vanishing $\Lambda$ [35 37]. It is evident that for $d=3$, Eq. (13) reduces to the HEE obtained by Diaz [80]. As a result, we have obtained a global form of HEE for arbitrary dimensions $(d \geq 3)$ in Eq. (13).

\section{STRUCTURE PROPERTIES OF NEUTRON STAR}

\section{A. Equation of state of neutron star matter}

Obtaining equation of state for neutron stars enables one to study its properties. The interior region of the neutron star is a mixture of neutrons, protons, electrons and muons. These constituents are in charge neutrality and beta equilibrium conditions (beta-stable matter) [81]. Recently, the microscopic constrained variational calculations based on the cluster expansion of the energy functional has been employed to obtain the equation of state of neutron star matter [56, 82, 83]. The two-nucleon potentials which are used in these studies are the modern Argonne AV18 [84] and charged dependent Reid-93 [85]. One of the advantages of these methods is that there is no need for any free parameter in formalism, and they result into a good convergence. Also, the results in this method are more accurate comparing to other semi-empirical parabolic approximation methods. This is due to a microscopic computation of asymmetry energy which is carried on for the asymmetric nuclear matter calculations. In fact, it is necessary to have a microscopic calculation with the modern nucleon-nucleon potentials which is isospin projection $\left(T_{z}\right)$ dependant [86]. Here, we are interested in using the lowest order constrained variational (LOCV) method with the AV18 potential [82, 83] for calculating the modern equation of state for neutron star matter and investigating some physical properties of neutron star structure. We use the LOCV method to calculate the energy of our system. The LOCV method is a useful tool for determination of the properties of neutron, nuclear and asymmetric nuclear matter at finite and zero temperature [87 91]. It is notable that, the LOCV method is a fully self-consistent formalism and it does not bring any free parameters into calculation. It employs a normalization constraint to keep the higher order term as small as possible [91, 92]. The functional minimization procedure represents an enormous computational simplification over unconstrained methods that attempt to go beyond lowest order.

A suitable trial many-body wave function is

$$
\psi=F \phi,
$$




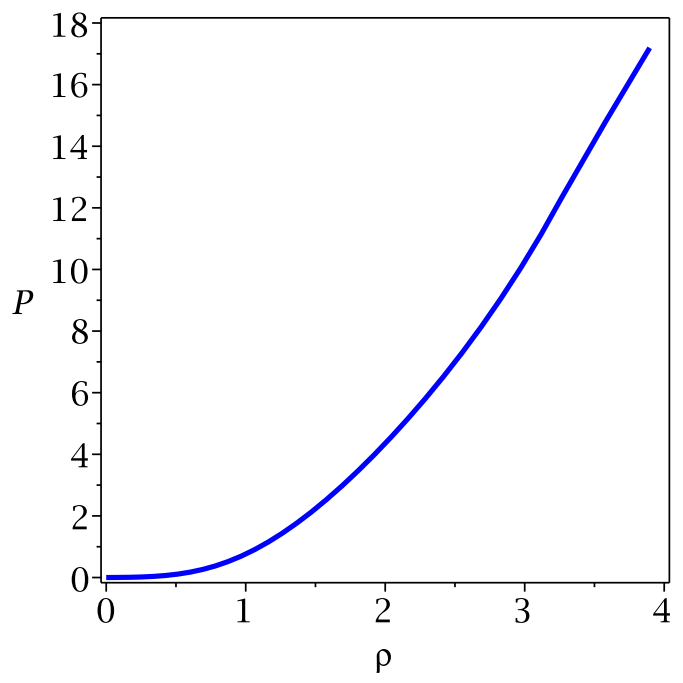

FIG. 1: Equation of state of neutron star matter (pressure, $P\left(10^{35} \mathrm{erg} / \mathrm{cm}^{3}\right)$ versus density, $\left.\epsilon\left(10^{15} \mathrm{gr} / \mathrm{cm}^{3}\right)\right)$.

where $\phi$ is the uncorrelated ground-state wave function of $N$ independent neutrons, and $F$ is a proper $N$-body correlation function. Applying Jastrow approximation [93], $F$ is rewritten by

$$
F=S \prod_{i>j} f(i j)
$$

where $S$ is a symmetrizing operator. Also, we consider a cluster expansion of the energy functional up to the two-body term

$$
E([f])=\frac{1}{N} \frac{\langle\psi|H| \psi\rangle}{\langle\psi \mid \psi\rangle}=E_{1}+E_{2},
$$

The energy per particle up to the two-body term is

$$
E([f])=E_{1}+E_{2},
$$

where $E_{1}=\sum_{i=+,-} \frac{3}{5} \frac{\hbar^{2} k_{F}^{(i)}{ }^{2}}{2 m} \frac{\epsilon^{(i)}}{\epsilon}$ and $E_{2}=\frac{1}{2 N} \sum_{i j}\langle i j|\nu(12)| i j-j i\rangle$ are one-body and two-body energy term, respectively. The operator $\nu(12)$ has been given in Ref. [94] (see Refs. [86, 95, 96] for more details). Our obtained equation of state of neutron star matter is shown in Fig. 1

In order to obtain more details for equation of state of neutron star matter introduced in this paper, we study both stability and energy conditions in the following subsections.

\section{Stability}

In order to investigate the stability of the introduced equation of state for a physically acceptable model, one expects that, the speed of sound $(v)$ be less than the speed of light $(c)$ [97, 98]. This stability condition can be written in the following explicit form

$$
0 \leq v^{2}=\left(\frac{d P}{d \epsilon}\right) \leq c^{2}
$$

By using the above condition and Fiq. 1, and comparing them with diagrams related to sound speed-radius relationship plotted in Ref. [99] (see Fig. 2 in Ref. [99], for more details), it is evident that this equation of state satisfies the inequality $0 \leq v^{2} \leq c^{2}$. Therefore, the introduced equation of state is stable. 
TABLE I: Energy conditions for neutron stars.

\begin{tabular}{|c|c|c|c|c|c|}
\hline$\epsilon_{0}\left(10^{9} \mathrm{~kg} / \mathrm{cm}^{3}\right)$ & $P_{0}\left(10^{9} \mathrm{~kg} / \mathrm{cm}^{3}\right)$ & $N E C$ & $W E C$ & $S E C$ & $D E C$ \\
\hline 3895.99 & 1910.39 & $\checkmark$ & $\checkmark$ & $\checkmark$ & $\checkmark$ \\
\hline
\end{tabular}

\section{Energy Conditions}

One of interesting properties of the mentioned equations of state is related to its central density. It is notable that the obtained central density of this equations of state is about $3.9 \times 10^{15} \mathrm{~g} \mathrm{~cm}^{-3}$ and this density is larger than the normal nuclear density $\epsilon_{0}=2.7 \times 10^{14} \mathrm{~g} \mathrm{~cm}^{-3}[100]$.

Now, we want to study the energy conditions. In this regard, we investigate null energy condition (NEC), weak energy condition (WEC), strong energy condition (SEC) and dominant energy condition (DEC) at the center of the neutron stars. These energy conditions can be written in the following explicit forms

$$
\begin{aligned}
N E C & \rightarrow P_{c}+\epsilon_{c} \geq 0, \\
W E C & \rightarrow P_{c}+\epsilon_{c} \geq 0, \quad \& \quad \epsilon_{c} \geq 0, \\
S E C & \rightarrow P_{c}+\epsilon_{c} \geq 0, \quad \& 3 P_{c}+\epsilon_{c} \geq 0, \\
D E C & \rightarrow \epsilon_{c}>\left|P_{c}\right|,
\end{aligned}
$$

where $P_{c}$ and $\epsilon_{c}$ are the pressure and density at the center of neutron star $(r=0)$, respectively. Considering Fig. 1 and the above conditions (20)-(23), our results are presented in Table 1 .

According to the Table I, we observe that all the energy conditions are satisfied. Therefore, the equation of state of neutron star matter introduced in this paper are suitable and consistent with all versions of energy conditions.

The equation of state of neutron star matter satisfied both stability and energy conditions. Now, we focus on investigation of maximum mass and radius for the neutron stars in Einstein- $\Lambda$ gravity.

\section{B. Properties of neutron stars in Einstein- $\Lambda$ gravity}

Considering the maximum gravitational mass of a neutron star for stability against collapse into a black hole, one is able to recognize differences between neutron stars and black holes. In other words, there is a critical maximum mass in which for smaller than such mass, degenerate pressure originated from the nucleons prevents an object from becoming a black hole [81]. Therefore, obtaining the maximum gravitational mass of neutron stars is of great importance in astrophysics. Due to many errors in direct ways of measuring the neutron star mass by observations of the X-ray pulsars and X-ray bursts, one is not able to obtain an accurate mass for these stars. On the other hand, using the binary radio pulsars [101 104], one may obtain highly accurate results for the mass of neutron stars.

Now, by employing the equation of state of neutron star matter presented in Fig. 1 and numerical approach for integrating the HEE obtained in Eq. (13), we obtain the maximum mass of neutron stars. The neutron star mass and radius depend on the central mass density $\left(\epsilon_{c}\right)$. We can consider the boundary conditions $P(r=0)=P_{c}$ and $m(r=0)=0$, and integrate Eq. (13) outward to a radius $r=R$ in which $P$ vanishes for selecting an $\epsilon_{c}$. This leads to the neutron star radius $R$ and mass $M=m(R)$.

In Ref. [99], the Einstein gravity without cosmological constant has been investigated, and maximum mass of neutron stars has been obtained using the modern equations of state of neutron star matter derived from microscopic calculations. It was shown that the maximum mass of neutron star is about $1.68 M_{\odot}$. In the present paper, we are going to consider the cosmological constant and obtain the maximum mass for neutron stars by employing the modern equations of state and study other properties of these stars. Our results are presented in Tables II and II.

The value of the cosmological constant and its fine tuning is open questions for scientists. In addition, its consistent values in various scenarios are different and therefore, it is allowed to regard it as a free parameter. On the other hand, from the standpoint of cosmology its order of magnitude may be approximately $10^{-52} \mathrm{~m}^{-2}$. Such order of magnitude may be valid in a special cosmological model with large scale structure. Meanwhile, in order to regard the effects of $\Lambda$ in local scales (such as near the black holes, neutron stars and other massive objects), it is not necessary to follow the fine tuning values of $\Lambda$ is large scales. Here, we regard the value $10^{-52} \mathrm{~m}^{-2}$ and also other values (at least as a toy model) to obtain the effects of cosmological constant on the neutron star structure. According to our results, the 
TABLE II: Einstein- $\Lambda$ gravity for 4-dimensions.

\begin{tabular}{cccccccc}
\hline \hline$\Lambda\left(m^{-2}\right)$ & $\frac{M_{\max }}{\left(M_{\odot}\right)}$ & $R(\mathrm{~km})$ & $R_{S c h}(\mathrm{~km})$ & $\bar{\epsilon}\left(10^{15} \mathrm{~g} \mathrm{~cm}^{-3}\right)$ & $\eta\left(10^{-1}\right)$ & $z$ & $M_{B B}\left(M_{\odot}\right)$ \\
\hline \hline 0 & 1.68 & 8.42 & 4.95 & 1.34 & 5.88 & 0.56 & 2.54 \\
\hline $1.00 \times 10^{-50}$ & 1.68 & 8.42 & 4.95 & 1.34 & 5.88 & 0.56 & 2.54 \\
\hline $1.00 \times 10^{-52}$ & 1.68 & 8.42 & 4.95 & 1.34 & 5.88 & 0.56 & 2.54 \\
\hline $1.00 \times 10^{-54}$ & 1.68 & 8.42 & 4.95 & 1.34 & 5.88 & 0.56 & 2.54 \\
\hline \hline
\end{tabular}

TABLE III: Einstein- $\Lambda$ gravity for 4 -dimensions.

\begin{tabular}{cccccccc}
\hline \hline$\Lambda\left(\mathrm{m}^{-2}\right)$ & $\frac{M_{\max }}{\left(M_{\odot}\right)}$ & $R(\mathrm{~km})$ & $R_{S c h}(\mathrm{~km}) \bar{\epsilon}\left(10^{15} \mathrm{~g} \mathrm{~cm}^{-3}\right)$ & $\eta\left(10^{-1}\right)$ & $z$ & $M_{B B}\left(M_{\odot}\right)$ \\
\hline \hline $1.00 \times 10^{-11}$ & 0.78 & 6.65 & 2.30 & 1.26 & 3.46 & 0.24 & 2.00 \\
\hline $5.00 \times 10^{-12}$ & 1.12 & 7.47 & 3.30 & 1.27 & 4.42 & 0.34 & 2.25 \\
\hline $1.00 \times 10^{-12}$ & 1.56 & 8.25 & 4.60 & 1.32 & 5.57 & 0.50 & 2.49 \\
\hline $5.00 \times 10^{-13}$ & 1.62 & 8.34 & 4.77 & 1.33 & 5.72 & 0.53 & 2.51 \\
\hline $1.00 \times 10^{-13}$ & 1.67 & 8.40 & 4.92 & 1.34 & 5.86 & 0.55 & 2.53 \\
\hline $1.00 \times 10^{-14}$ & 1.68 & 8.42 & 4.95 & 1.34 & 5.88 & 0.56 & 2.54 \\
\hline $1.00 \times 10^{-15}$ & 1.68 & 8.42 & 4.95 & 1.34 & 5.88 & 0.56 & 2.54 \\
\hline \hline
\end{tabular}

cosmological constant has no significant effect when we consider its value identical to $10^{-52} \mathrm{~m}^{-2}$ (see Table II , for more details). Therefore, in order to examine its effects, we should consider a (an ad hoc) model in which its value is less than $10^{-14} \mathrm{~m}^{-2}$.

Based on Table III considering the cosmological constant as a positive quantity, the maximum mass of neutron star decreases as $\Lambda$ increases. In other words, the maximum mass of neutron stars with the positive values of cosmological constant are in the range $M_{\max } \leq 1.68 M_{\odot}$. Also, when we consider the cosmological constant to be very small $\left(\Lambda \leq 10^{-14}\right)$, the maximum mass reduces to results obtained in the Einstein gravity [99]. In other words, for $\Lambda \leq 10^{-14}$ this constant does not affect the maximum mass and other properties. So, considering the obtained value for the cosmological constant from the cosmological perspective $\left(\Lambda \simeq 10^{-52} m^{-2}\right)$, this quantity does not affect the structure of neutron stars.

We plot diagrams related to the maximum mass versus central mass density (radius) in Figs. 22and 3, The variation of maximum mass versus the cosmological constant is also shown in Fig. 4 for $\Lambda>0$.

As we can see, mass of neutron star versus radius changes when the $\Lambda$ increases. Indeed, one of interesting results of this paper is related to change in diagrams of mass versus radius, so that by increasing $\Lambda$, these diagrams change from ordinary shapes of the neutron stars to those of quark stars (see Fig. 3). In other words, by increasing $\Lambda$, we encounter with a change of state (phase transition) of neutron star to the quasi quark star. On the other hand, we consider negative values of the cosmological constant and plot the density and pressure versus radius of neutron star. As one can see, the maximum and minimum values belong to the center and surface of neutron star, respectively (see Figs. 5 and 6), but the behavior of diagrams related to mass-radius relation of these stars are not logical (see Fig. (7). In other words, Einstein gravity with the negative values of the cosmological constant are not a suitable theory for explaining the neutron stars with the mentioned equation of state.

Our results show that, by decreasing the cosmological constant $\left(\Lambda<10^{-14} \mathrm{~m}^{-2}\right)$, the maximum mass and also the radius of this star are not modified. In other words, the cosmological constant does not significantly affect the maximum and radius of neutron stars for $\Lambda<10^{-14} \mathrm{~m}^{-2}$. As a result of this paper, when the value of the cosmological constant is about $10^{-52} \mathrm{~m}^{-2}$, this constant does not play a sensible role in the structure of neutron stars, but by considering larger values for it (about $\Lambda>10^{-14} \mathrm{~m}^{-2}$ ), the maximum mass and its radius are modified (reduced).

In the following, we investigate other properties of neutron stars by considering the cosmological constant such as; the Schwarzschild radius, average density, compactness, Buchdahl-Bondi bound and dynamical stability. 


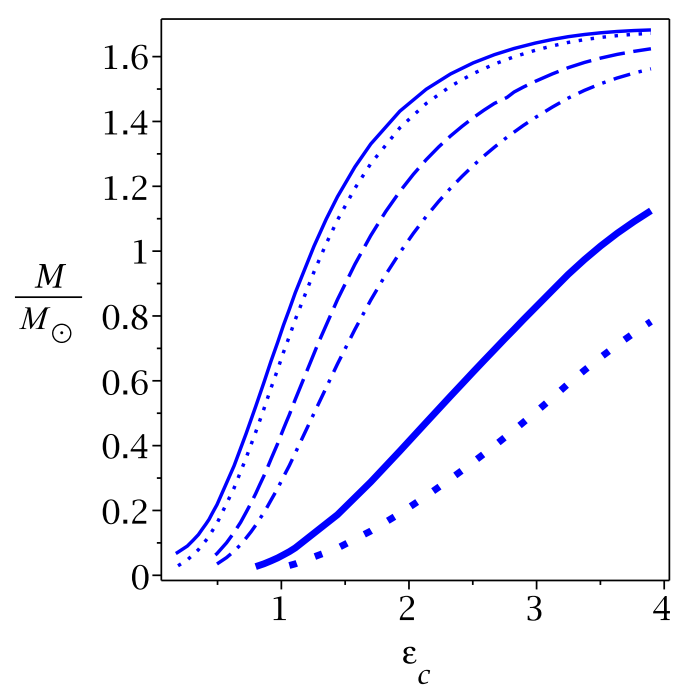

FIG. 2: Maximum mass of neutron star versus central mass density $\epsilon_{c}\left(10^{15} \mathrm{gr} / \mathrm{cm}^{3}\right)$, for $\Lambda=1 \times 10^{-14}$ (continuous line), $\Lambda=1 \times 10^{-13}$ (dotted line), $\Lambda=5 \times 10^{-13}$ (dashed line), $\Lambda=1 \times 10^{-12}$ (dashed-dotted line), $\Lambda=5 \times 10^{-12}$ (bold line) and $\Lambda=1 \times 10^{-11}$ (bold-dotted line).

\section{Schwarzschild Radius}

Now, we obtain the Schwarzschild radius for the obtained masses in Einstein- $\Lambda$ gravity in the following form

$$
R_{S c h}=\frac{\left[\left(3 G M+\sqrt{\frac{c^{4}}{\Lambda}+9 G^{2} M^{2}}\right) \Lambda^{2} c\right]^{1 / 3}}{\Lambda c}-\frac{c}{\left[\left(3 G M+\sqrt{\frac{c^{4}}{\Lambda}+9 G^{2} M^{2}}\right) \Lambda^{2} c\right]^{1 / 3}},
$$

where $R_{S c h}$ is the Schwarzschild radius, $G=6.67 \times 10^{-11} \mathrm{Nm}^{2} / \mathrm{kg}^{2}$ and $\mathrm{c}=3 \times 10^{8} \mathrm{~m} / \mathrm{s}$. It is notable that, by applying the cosmological constant to the Einstein gravity, the Schwarzschild radius modified and also, considering $\Lambda=0$ in Eq. (??), it reduces to the Schwarzschild radius in Einstein gravity as 105]

$$
R_{S c h}=\frac{2 G M}{c^{2}} \text {. }
$$

To find the Schwarzschild radius of neutron stars, we use of Eq. (??). The results show that increasing the cosmological constant and decreasing the maximum mass of neutron stars, the Schwarzschild radius decreases (see Table III for more details).

\section{Average Density}

The average density of the neutron stars in 4-dimensions can be written as the following form

$$
\bar{\epsilon}=\frac{3 M}{4 \pi R^{3}},
$$

where the results for various the cosmological constant are presented in Table III

\section{Compactness}

The compactness of a spherical object may be defined as the ratio of the Schwarzschild radius to the radius of object as

$$
\eta=\frac{R_{S c h}}{R},
$$



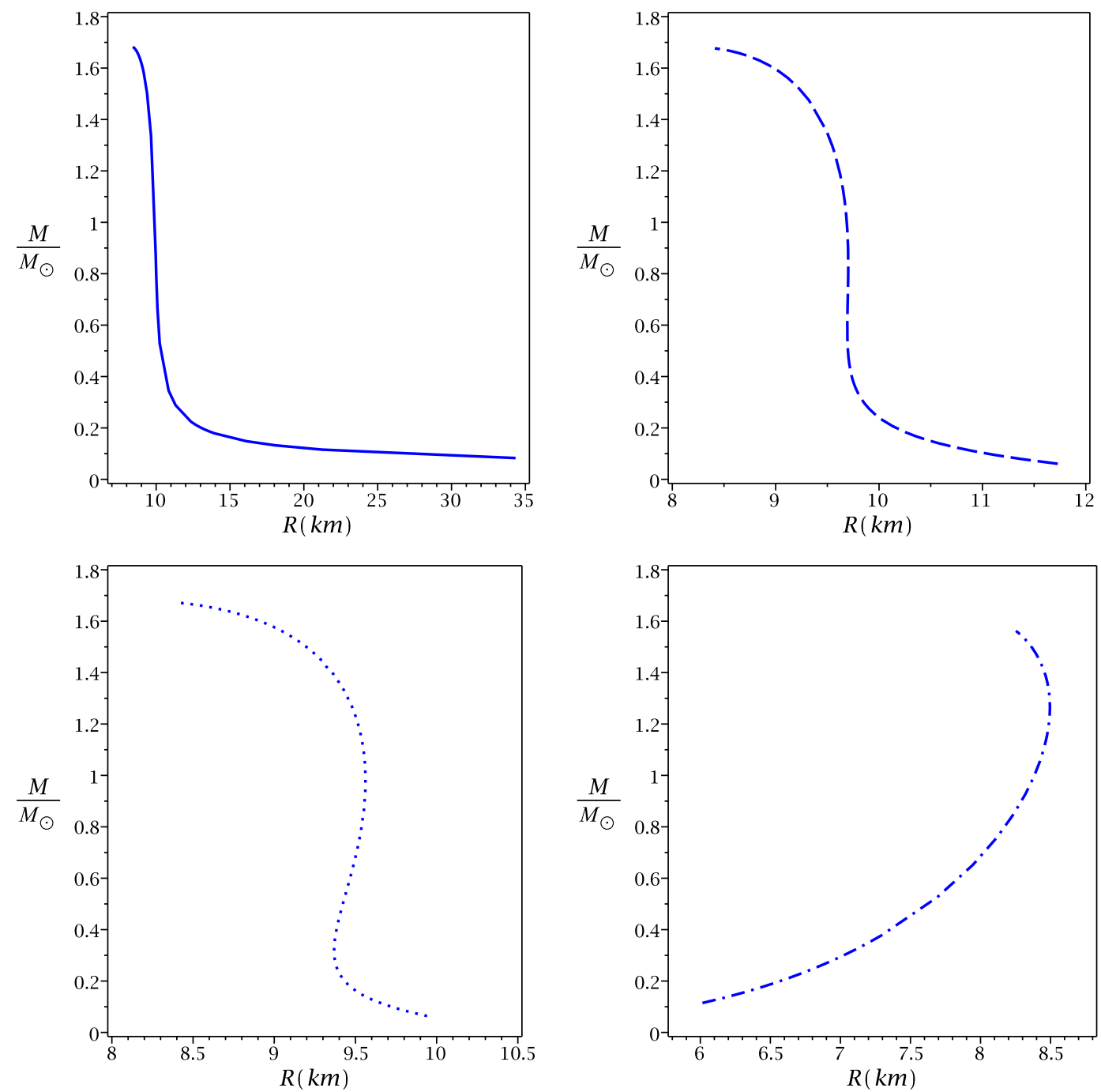

FIG. 3: Maximum mass of neutron star versus radius for $\Lambda=1 \times 10^{-15}$ (continuous line), $\Lambda=6 \times 10^{-14}$ (dashed line), $\Lambda=1 \times 10^{-13}$ (dotted line) and $\Lambda=1 \times 10^{-12}$ (dashed-dotted line).

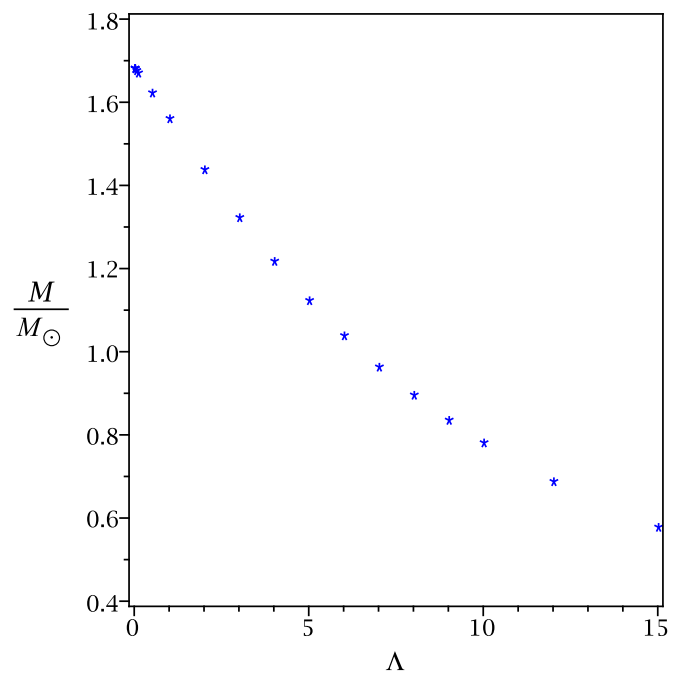

FIG. 4: Maximum mass of neutron star versus cosmological constant $\left(\times 10^{-12}\right)$ in 4 -dimensions. 


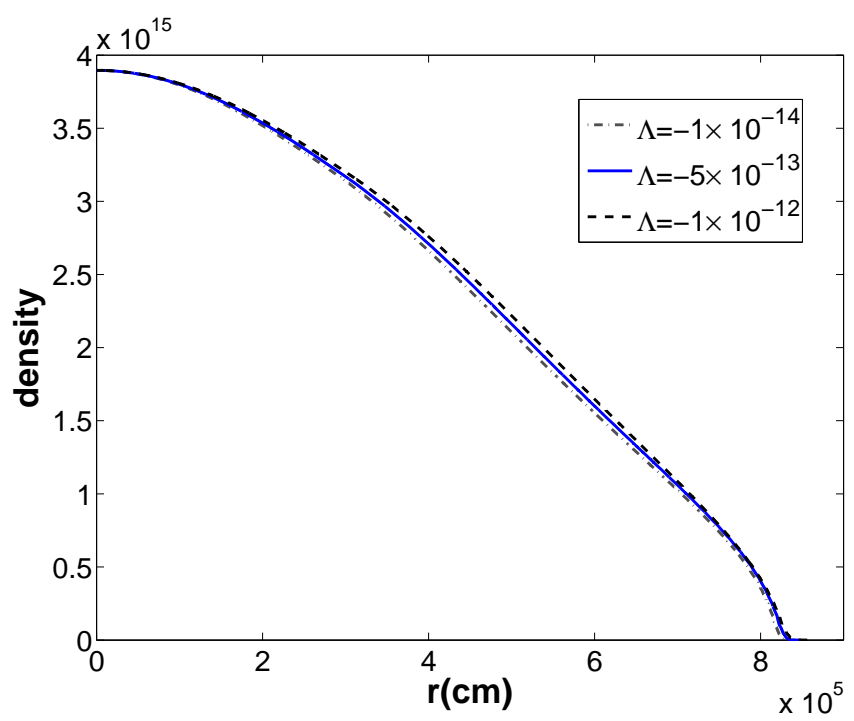

FIG. 5: Density of neutron star versus radius.

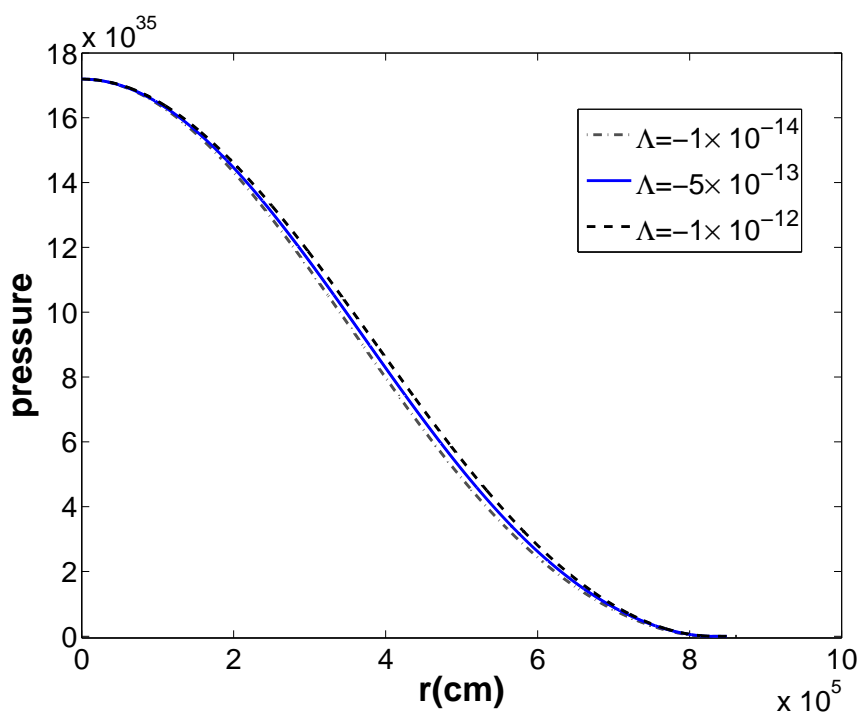

FIG. 6: Pressure of neutron star versus radius.

which may be indicated as the strength of gravity. We obtain the values of $\eta$ in the Einstein- $\Lambda$ gravity and find that increasing $\Lambda$ leads to decreasing the strength of gravity.

\section{The Gravitational Redshift}

Using the equation (12), we obtain the gravitational redshift in 4-dimensions for this gravity as

$$
z=\frac{1}{\sqrt{1-\frac{2 G M}{c^{2} R}-\frac{\Lambda}{3} R^{2}}}-1 .
$$

As one can see, decreasing $\Lambda$ leads to increasing the gravitational redshift (see Table III). The maximum value of the gravitational redshift is about 0.56. According to Table III, one finds variation of $\Lambda$ changes the gravitational redshift, drastically. This is a way for adjusting the values of $\Lambda$ based on observational evidences. 

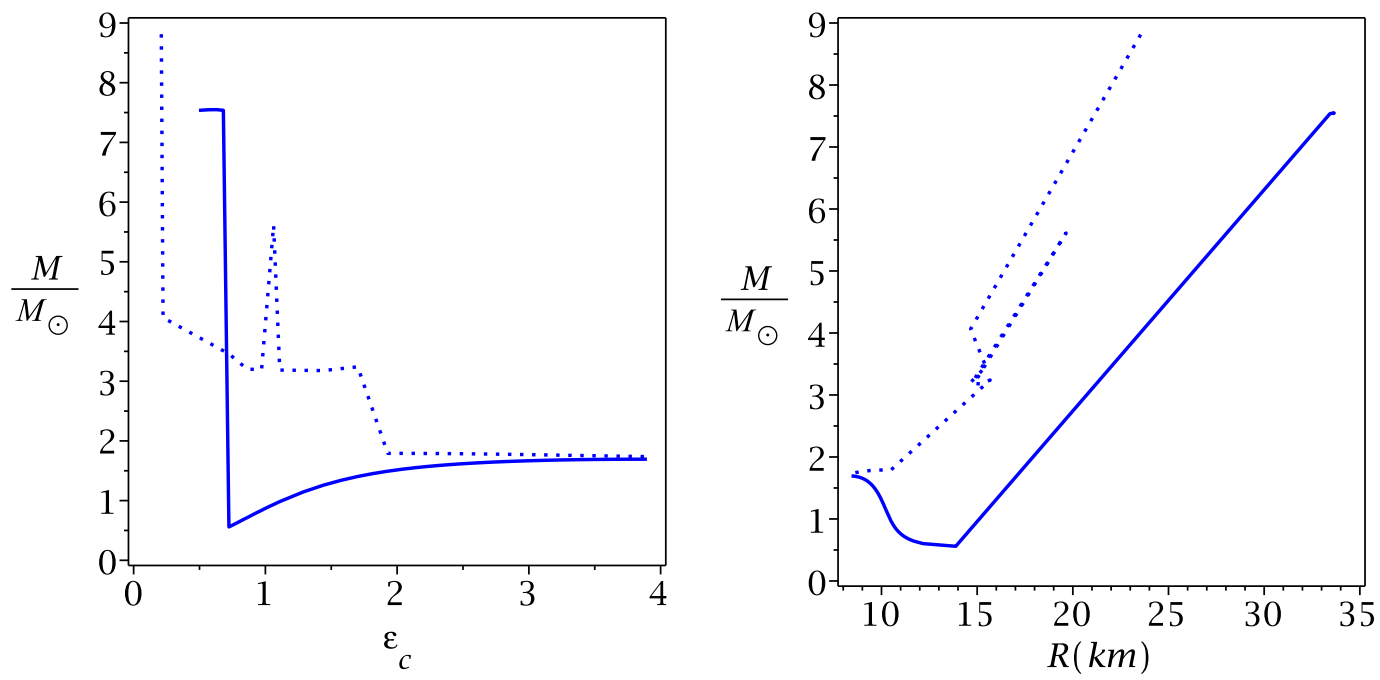

FIG. 7: Maximum mass of neutron star versus central mass density $\epsilon_{c}\left(10^{15} \mathrm{gr} / \mathrm{cm}^{3}\right)$, (left panel) and radius (right panel) for $\Lambda=-9 \times 10^{-14}$ (continuous line) and $\Lambda=-5 \times 10^{-13}$ (dotted line).

\section{Buchdahl-Bondi Bound}

According to Buchdahl-Bondi bound [106 108], for a static spherically symmetric perfect fluid sphere, maximum permitted $M-R$ ratio should be $M \leq \frac{4 c^{2}}{9 G} R$. For the Einstein- $\Lambda$ gravity this bound modified as [109]

$$
M \leq M_{B B}
$$

where $M_{B B}=\frac{2 c^{2}}{9 G} R-\frac{\Lambda c^{2}}{3 G} R^{3}+\frac{2 c^{2}}{9 G} R \sqrt{1+3 \Lambda R^{2}}$. Using the above condition, we investigate the stability condition of neutron stars in Einstein- $\Lambda$ gravity. The calculations are presented in Table III The results show that obtained maximum mass of neutron stars for all values obtained in Table III are stable.

\section{Dynamical Stability}

The dynamical stability of the stellar model against the infinitesimal radial adiabatic perturbation was introduced by Chandrasekhar in Ref. [110]. Next, this stability condition was developed and applied to astrophysical cases by many authors [111 114]. The adiabatic index $(\gamma)$ is defined as

$$
\gamma=\frac{\epsilon c^{2}+P}{c^{2} P} \frac{d P}{d \epsilon} .
$$

It is notable that, in order to have the dynamical stability, $\gamma$ should be larger than $\frac{4}{3}\left(\gamma>\frac{4}{3}=1.33\right)$ everywhere within the isotropic stars. So, we plot a diagram related to $\gamma$ versus radius for different values of the cosmological constant in Fig. 8. As one can see, this stellar model is stable against the radial adiabatic infinitesimal perturbations.

On the other hand, in order to present more investigations for the inside of neutron stars in Einstein- $\Lambda$ gravity, we plot density and pressure versus radius of this star in Figs. 9 and 10. These figures show that density (pressure) values have a maximum and a minimum in the center and surface of neutron star, respectively, as expected.

\section{CLOSING REMARKS}

In the first part of this paper, we have considered a spherical symmetric metric and extracted the hydrostatic equilibrium equation of stars for $d$-dimensional Einstein- $\Lambda$ gravity $(d \geq 3)$. Then, we have investigated the maximum mass of neutron stars by using the modern equations of state of neutron star matter derived from microscopic calculations. The results showed that the cosmological constant affects the maximum mass of neutron stars. In other 


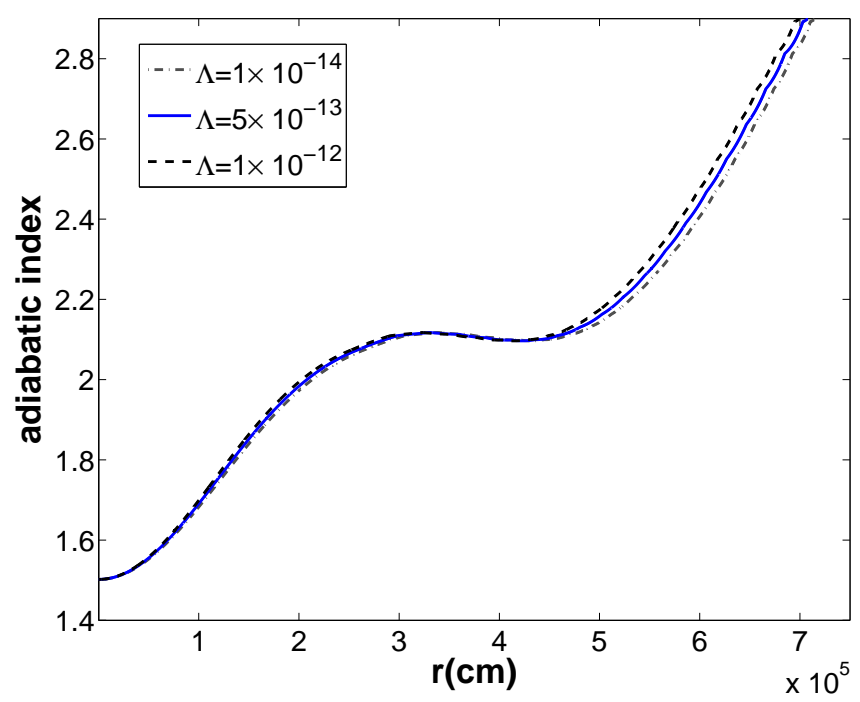

FIG. 8: Adiabatic index of neutron star versus radius.

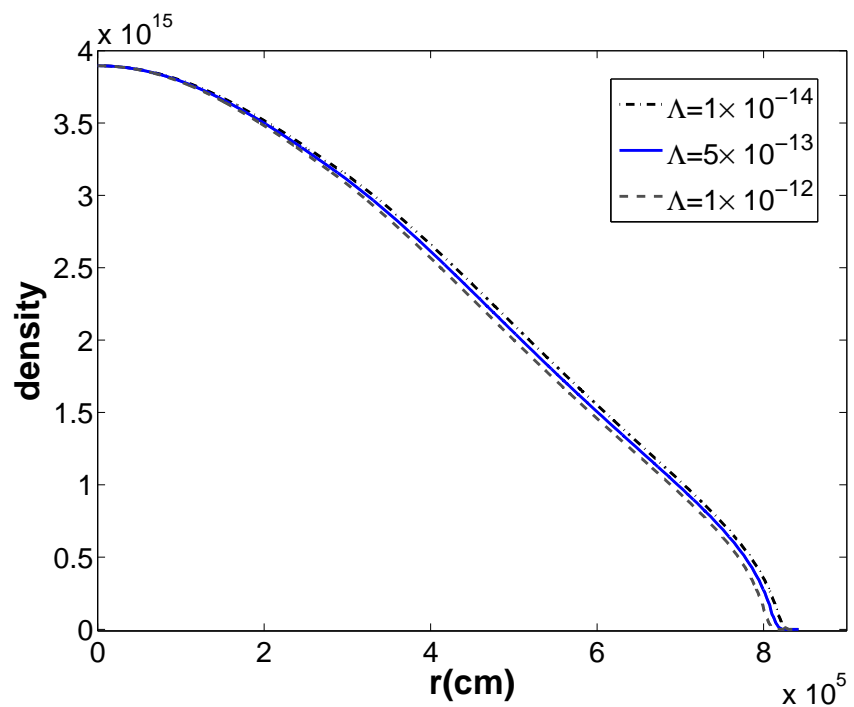

FIG. 9: Density of neutron star versus radius.

words, considering the $\Lambda>0$, the maximum mass for neutron stars decreases when $\Lambda$ increases. In this case, maximum mass of neutron stars is in the range $M_{\max } \leq 1.68 M_{\odot}$.

One of the interesting results obtained in this paper is the behavior of diagrams of mass versus radius of the neutron stars. By increasing $\Lambda$, these diagrams showed a transition from the neutron star to the quasi quark star. On the other hand, considering $\Lambda<0$, the behavior of diagrams related to mass versus radius (central mass density) of neutron stars is not logical, so the Einstein gravity with the negative values of cosmological constant can not explain the existence of neutron stars. In order to investigate the interior of neutron stars in Einstein- $\Lambda$ gravity in more details, we have plotted density and pressure versus radius of this star. The results showed that the density and pressure were maximum and minimum of values at the central and surface of neutron stars, respectively.

Our results showed that, when the approximate value of the cosmological constant was $10^{-52} \mathrm{~m}^{-2}$, this constant did not play a sensitive role in the structure of neutron stars, but by taking larger values for it (about $\Lambda>10^{-14} \mathrm{~m}^{-2}$ ), the maximum mass and its radius were reduced. Another interesting result of this paper is related to the effect of cosmological constant on the so-called gravity strength. We have shown that when the positive values of the cosmological constant increases, the strength of gravity decreases, and this effect leads to decreasing the maximum mass of neutron stars.

Finally, we have studied the stability condition for these stars using Buchdahl-Bondi bound and found that the 


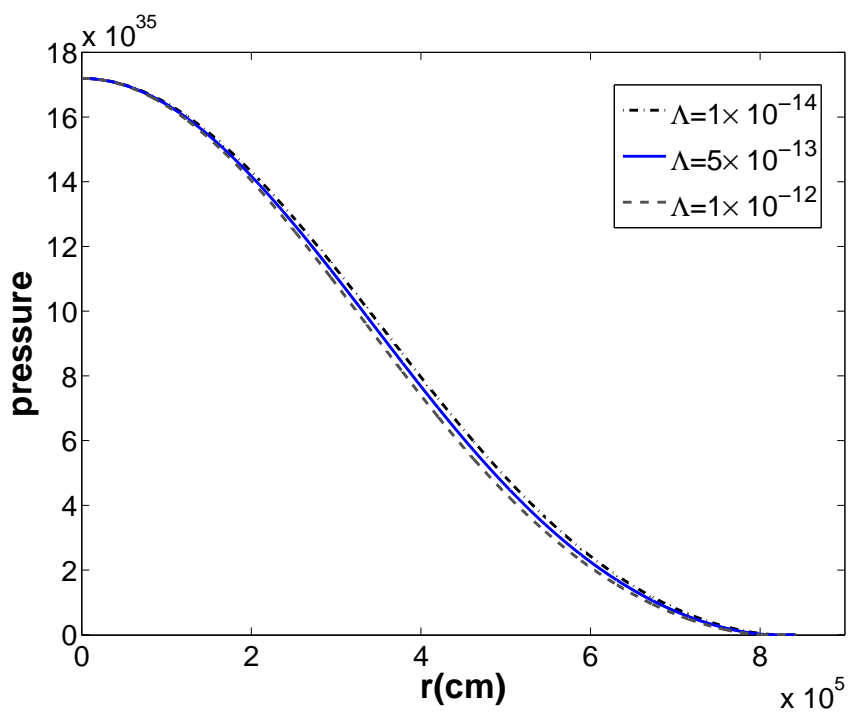

FIG. 10: Pressure of neutron star versus radius.

obtained maximum mass of neutron stars for all values obtained in Table III were stable. Then, considering adiabatic index, we showed that these stars were stable against the radial adiabatic infinitesimal perturbations.

\section{acknowledgements}

We would like to thank the anonymous referee for his/her valuable comments. We also wish to thank Shiraz University Research Council. GHB wishes to thank the Center for Excellence in Astronomy and Astrophysics (CEAARIAAM) for financial support. BE acknowledges S. Panahiyan for helpful discussions. This work has been supported financially by Research Institute for Astronomy and Astrophysics of Maragha.

[1] S. Perlmutter et al., Astrophys. J. 517, 565 (1999).

[2] S. Perlmutter, M. S. Turner and M. White, Phys. Rev. Lett. 83, 670 (1999).

[3] A. G. Riess et al., Astrophys. J. 607, 665 (2004).

[4] D. Lovelock. J. Math. Phys. 12, 498 (1971).

[5] D. Lovelock. J. Math. Phys. 13, 874 (1972).

[6] M. H. Dehghani and S. H. Hendi, Gen. Relativ. Gravit. 41, 1853 (2009).

[7] S. H. Hendi, B. Eslam Panah and S. Panahiyan, Phys. Rev. D 91, 084031 (2015).

[8] P. Brax and C. van de Bruck, Class. Quantum Gravit. 20, R201 (2003).

[9] L. A. Gergely, Phys. Rev. D 74, 024002 (2006).

[10] M. Demetrian, Gen. Relativ. Gravit. 38, 953 (2006).

[11] C. Brans and R. H. Dicke, Phys. Rev. 124, 925 (1961).

[12] R. G. Cai and Y. S. Myung, Phys. Rev. D 56, 3466 (1997).

[13] T. P. Sotiriou, Class. Quantum Gravit. 23, 5117 (2006).

[14] K. I. Maeda and Y. Fujii, Phys. Rev. D 79, 084026 (2009).

[15] S. H. Hendi and R. Katebi, Eur. Phys. J. C 72, 1 (2012).

[16] T. Harada, Phys. Rev. D 57, 4802 (1998).

[17] D. D. Doneva, S. S. Yazadjiev, N. Stergioulas, K. D. Kokkotas and T. M. Athanasiadis, Phys. Rev. D 90, 044004 (2014).

[18] M. Ponce, C. Palenzuela, E. Barausse and L. Lehner, Phys. Rev. D 91, 084038 (2015).

[19] A. Cisterna, T. Delsate and M. Rinaldi, Phys. Rev. D 92, 044050 (2015).

[20] C. Palenzuela and S. Liebling, Phys. Rev. D 93, 044009 (2016).

[21] J. C. C. de Souza and V. Faraoni. Class. Quantum Gravit. 24, 3637 (2007).

[22] G. Cognola, E. Elizalde, S. Nojiri, S. D. Odintsov, L. Sebastiani, and S. Zerbini. Phys. Rev. D, 77, 046009 (2008)

[23] S. Nojiri and S. D. Odintsov, Phys. Rept. 505, 59 (2011).

[24] N. R. Napolitano, S. Capozziello, A. J. Romanowsky, M. Capaccioli and C. Tortora, Astrophys. J. 748, 87 (2012).

[25] S. H. Hendi, R. B. Mann, N. Riazi and B. Eslam Panah, Phys. Rev. D 86, 104034 (2012).

[26] M. Lubini, C. Tortora, J. Naf, Ph. Jetzer and S. Capozziello, Eur. Phys. J. C 71, 1834 (2011).

[27] A. Cooney, S. D. Deo and D. Psaltis, Phys. Rev. D 82, 064033 (2010). 
[28] S. H. Hendi, B. Esam Panah and S. M. Mousavi, Gen. Relativ. Gravit. 44, 835 (2012).

[29] S. H. Hendi, B. Eslam Panah and C. Corda, Can. J. Phys. 92, 76 (2014).

[30] S. Capozziello, M. D. Laurentis, R. Farinelli and S. D. Odintsov, Phys. Rev. D 93, 023501 (2016).

[31] C. Gao and Y. G. Shen, Gen. Relativ. Gravit. 48, 1 (2016).

[32] P. J. E. Peebles and B. Ratra, Rev. Mod. Phys. 75, 559 (2003).

[33] T. Padmanabhan, Phys. Rept. 380, 235 (2003).

[34] J. A. Frieman, M. S. Turner and D. Huterer, Ann. Rev. Astron. Astrophys. 46, 385 (2008).

[35] R. C. Tolman, Proc. Nat. Acad. Sc. 20, 169 (1934).

[36] R. C. Tolman, Phys. Rev. 55, 364 (1939).

[37] J. R. Oppenheimer and G. M. Volkoff, Phys. Rev. 55, 374 (1939).

[38] N. Yunes and M. Visser, Int. J. Mod. Phys. A 18, 3433 (2003).

[39] R. R. Silbar and S. Reddy, Am. J. Phys. 72, 892 (2004).

[40] G. Narain, J. Schaffner-Bielich and I. N. Mishustin, Phys. Rev. D 74, 063003 (2006).

[41] G. H. Bordbar, M. Bigdeli and T. Yazdizade, Int. J. Mod. Phys. A 21, 5991 (2006).

[42] P. Boonserm, M. Visser and S. Weinfurtner, Phys. Rev. D 76, 044024 (2007).

[43] X. Li, F. Wang and K. S. Cheng, JCAP 10, 031 (2012).

[44] A. M. Oliveira, H. E. S. Velten, J. C. Fabris and I. G. Salako, Eur. Phys. J. C 74, 3170 (2014).

[45] X. T. He, F. J. Fattoyev, B. A. Li and W. G. Newton, Phys. Rev. C 91, 015810 (2015).

[46] T. Wiseman, Phys. Rev. D 65, 124007 (2002).

[47] H. Sotani, Phys. Rev. D 86, 124036 (2012).

[48] D. D. Doneva, S. S. Yazadjiev, N. Stergioulas and K. D. Kokkotas, Phys. Rev. D 88, 084060 (2013).

[49] N. Chamel, P. Haensel, J. L. Zdunik and A. F. Fantina, Int. J. Mod. Phys. E 22, 1330018 (2013).

[50] E. Barausse, C. Palenzuela, M. Ponce and L. Lehner, Phys. Rev. D 87, 081506(R) (2013).

[51] H. O. Silva, H. Sotani, E. Berti and M. Horbatsch, Phys. Rev. D 90, 124044 (2014).

[52] Y. Brihaye and J. Riedel, Phys. Rev. D 89, 104060 (2014).

[53] H. O. Silva, C. F. B. Macedo, E. Berti and L. C. B. Crispino, Class. Quantum Gravit. 32, 145008 (2015).

[54] A. Das, F. Rahaman, B. K. Guha and S. Ray, Astrophys. Space. Sci. 358, 36 (2015).

[55] K. V. Staykov, D. D. Doneva, S. S. Yazadjiev and K. D. Kokkotas, Phys. Rev. D 92, 043009 (2015).

[56] S. H. Hendi, G. H. Bordbar, B. Eslam Panah and M. Najafi, Astrophys. Space. Sci. 358, 30 (2015).

[57] S. H. Hendi, G. H. Bordbar, B. Eslam Panah and S. Panahiyan, JCAP 09, 013 (2016).

[58] T. Katsuragawa, S. Nojiri, S. D. Odintsov, M. Yamazaki, Phys. Rev. D 93, 124013 (2016).

[59] A. V. Astashenok, S. Capozziello and S. D. Odintsov, Astrophys. Space. Sci. 355, 341 (2015).

[60] A. V. Astashenok, S. Capozziello and S. D. Odintsov, JCAP 01, 001 (2015).

[61] G. Abbas, D. Momeni, M. Amir Ali, R. Myrzakulov and S. Qaisar, Astrophys. Space Sci. 357, 158 (2015).

[62] S. S. Yazadjiev, D. D. Doneva and K. D. Kokkotas, Phys. Rev. D 91, 084018 (2015).

[63] A. Savas Arapoglu, C. Deliduman and K. Yavuz Eksi, JCAP 07, 020 (2011).

[64] K. Zhou, Z. Y. Yang, D. C. Zou and R. H. Yue, Chin. Phys. B 21, 020401 (2012).

[65] K. Glampedakis, G. Pappas, H. O. Silva and E. Berti, Phys. Rev. D 92, 024056 (2015).

[66] T. Boyadjiev, P. Fiziev and S. Yazadjiev, Class. Quantum Gravit. 16, 2359 (1999).

[67] O. K. Vinayaraj and V. C. Kuriakose, arXiv:0802.1155.

[68] M. Heydari-Fard and H. R. Sepangi, JCAP 02, 029 (2009).

[69] S. Meyer, F. Pace and M. Bartelmann, Phys. Rev. D 86, 103002 (2012).

[70] A. V. Astashenok, S. Capozziello and S. D. Odintsov, JCAP 12, 040 (2013).

[71] M. Orellana, F. García, F. A. T. Pannia and G. E. Romero, Gen. Relativ. Gravit. 45, 771 (2013).

[72] J. D. V. Arbanil, J. P. S. Lemos and V. T. Zanchin, Phys. Rev. D 88, 084023 (2013).

[73] R. Goswami, A. M. Nzioki, S. D. Maharaj and S. G. Ghosh, Phys. Rev. D 90, 084011 (2014).

[74] J. P. S. Lemos, F. J. Lopes, G. Quinta and V. T. Zanchin, Eur. Phys. J. C 75, 76 (2015).

[75] D. Momeni, H. Gholizade, M. Raza and R. Myrzakulov, Int. J. Mod. Phys. A 30, 1550093 (2015).

[76] K. Glampedakis, G. Pappas, H. O. Silva and E. Berti, Phys. Rev. D 92, 024056 (2015).

[77] H. Velten, A. M. Oliveira and A. Wojnar, arXiv:1601.03000.

[78] M. B. Green and J. H. Schwarz, Phys. Lett. B 151, 21 (1985).

[79] P. Candelas, G. Horowitz, A. Strominger and E. Witten, Nucl. Phys. B 258, 46 (1985).

[80] A. A. G. Diaz, arXiv:1412.5620.

[81] S. Shapiro and S. Teukolsky, Black Holes, White Dwarfs and Neutron Stars. Wiley, New York (1983).

[82] G. H. Bordbar and N. Riazi, Astrophys. Space Sci. 282, 563 (2002).

[83] G. H. Bordbar, Int. J. Theor. Phys. 43, 399 (2004).

[84] R. B. Wiringa, V. Stoks and R. Schiavilla, Phys. Rev. C 51, 38 (1995).

[85] V. G. J. Stoks, R. A. M. Klomp, C. P. F. Terheggen and J. J. de Swart, Phys. Rev. C 49, 2950 (1994).

[86] G. H. Bordbar and M. Modarres, Phys. Rev. C 57, 714 (1998).

[87] M. Modarres and G. H. Bordbar, Phys. Rev. C 58, 2781 (1998).

[88] M. Modarres and J. M. Irvine, J. Phys. G: Nucl. Part. Phys. 5, 511 (1979).

[89] C. Howes, R. F. Bishop and J. M. Irvine, J. Phys. G: Nucl. Part. Phys. 4, 89 (1978).

[90] M. Modarres, J. Phys. G: Nucl. Part. Phys. 21, 351 (1995).

[91] J. C. Owen, R. F. Bishop and J. M. Irvine, Nucl. Phys. A 277, 45 (1977). 
[92] G. H. Bordbar and M. Modarres, J. Phys. G: Nucl. Part. Phys. 23, 1631 (1997).

[93] J. W. Clark, Prog. Part. Nucl. Phys. 2, 89 (1979).

[94] G. H. Bordbar, Z. Rezaei and A. Montakhab, Phys. Rev. C 83, 044310 (2011).

[95] G. H. Bordbar and M. Bigdeli, Phys. Rev. C 75, 045804 (2007).

[96] G. H. Bordbar and M. Bigdeli, Phys. Rev. C 77, 015805 (2008).

[97] L. Herrera, Phys. Lett. A 165, 206 (1992).

[98] H. Abreu, H. Hernandez and L. A. Nunes, Class. Quantum Gravit. 24, 4631 (2007).

[99] G. H. Bordbar and M. Hayati, Int. J. Mod. Phys. A 21, 1555 (2006).

[100] R. B. Wiringa, V. Fiks and A. Fabrocini, Phys. Rev. C 38, 1010 (1988).

[101] J. M. Weisberg and J. H. Taylor, Phys. Rev. Lett. 52, 1348 (1984).

[102] E. P. Liang, Astrophys. J. 304, 682 (1986).

[103] S. R. Heap and M. F. Corcoran, Astrophys. J. 387, 340 (1992).

[104] H. Quaintrell, Astron. Astrophys. 401, 313 (2003).

[105] K. Schwarzschild, translation by: S. Antoci and A. Loinger, arXiv:physics/9905030.

[106] H. A. Buchdahl, Phys. Rev. 116, 1027 (1959).

[107] H. Bondi, Proc. R. Soc. Lond. A 282, 303 (1964).

[108] H. A. Buchdahl, Astrophys. J. 146, 275 (1966).

[109] M. K. Mak, Peter N. Dobson, Jr., T. Harko, Mod. Phys. Lett. A 15, 2153 (2000).

[110] S. Chandrasekhar, Astrophys. J. 140, 417 (1964).

[111] J. M. Bardeen, K. S. Thonre and D. W. Meltzer, Astrophys. J. 145, 505 (1966).

[112] H. Kuntsem, MNRAS. 232, 163 (1988).

[113] M. K. Mak and T. Harko, Eur. Phys. J. C 73, 2585 (2013).

[114] M. Kalam, S. M. Hossein and S. Molla, arXiv:1510.07015. 